\title{
Treatment-Free Remission in Chronic Myeloid Leukemia Harboring Atypical BCR- ABL1 Transcripts
}

Keywords: TFR; Chronic myeloid leukemia; Atypical transcripts; digital PCR.

Citation: Dragani M., Petiti J., Rage-Cambrin G., E. Gottardi, Daraio F., Caocci G., Aguzzi C., Crisà E., Andreani G., Caciolli F., Fava C. Treatment-free remission in chronic myeloid leukemia harboring atypical BCR-ABL1 transcripts. Mediterr J Hematol Infect Dis 2020, 12(1): e2020066, DOI: http://dx.doi.org/10.4084/MJHID.2020.066

This is an Open Access article distributed under the terms of the Creative Commons Attribution License (https://creativecommons.org/licenses/by-nc/4.0), which permits unrestricted use, distribution, and reproduction in any medium, provided the original work is properly cited.

\section{To the editor.}

The majority of Philadelphia-positive chronic myeloid leukemia (CML) patients carry a $t(9 ; 22)$ translocation characterized by chromosomal breakpoints located on exon 13 or 14 of the $B C R$ gene and exon 2 of the $A B L 1$ gene (e13a2 or e14a2). This translocation generates a fusion gene whose epidemiology has been recently evaluated by the International BCR-ABL1 Study Group. ${ }^{1}$ It has been reported that the type of transcript influences the rate of complete cytogenetic response, the rate of major/deep molecular response, and the time needed to obtain major molecular response (MMR) during first-line imatinib or nilotinib treatment. Worldwide experiences also report inferior overall survival, leukemia related survival, progression-free survival and transformation-free survival in e13a2 patients, but this statement has not been confirmed in all studies. ${ }^{2-5}$

Type of transcript is of interest also in the field of treatment-free remission (TFR), which is the current goal of all hematologists who treat CML, although not all reports on discontinuation take into consideration this variable.

In rare cases of CML, breakpoints on chromosomes
9 and 22 occur in unusual regions, giving rise to atypical fusion transcripts. These transcripts, including e13a3, e14a3, e1a3, e19a2, e8a2, are not amplified by quantitative Real-Time PCR (RT-qPCR), which is the standardized and recommended method of molecular response evaluation. Current recommendations and guidelines consider the possibility to perform RT-qPCR on BCR-ABL1 as one of the criteria to meet to pursue tyrosine kinases inhibitors (TKI) stop both in clinical trials and in everyday practice as well. ${ }^{6,7}$

Nowadays, disease monitoring in atypical transcripts patients is performed routinely by non-quantitative Nested PCR, providing only an idea of their minimal residual disease (MRD) status.

Not having certainties about their biological behavior, due to their rarity, the lack of quantitative information about their molecular response automatically excludes patients with atypical transcripts from prospective protocols on TKI discontinuation.

We retrospectively collected seven patients with chronic-phase CML carrying rare atypical transcripts, identified by Sanger sequencing, ${ }^{8}$ who discontinued TKI for various reasons, such as severe comorbidities, toxicity, or patient request (Table 1).

Table 1. Patients' features.

\begin{tabular}{|c|c|c|c|c|c|c|}
\hline Patient & Transcript & $\begin{array}{c}\text { Time on TKI before } \\
\text { stop (months) }\end{array}$ & Treatment & $\begin{array}{c}\text { Duration of MMR } \\
\text { before stop (months) }\end{array}$ & Loss of MMR & TFR (months) \\
\hline 1 & $\mathrm{~b} 2 \mathrm{a} 3$ & 66 & Dasatinib & 48 & No & $55+$ \\
\hline 2 & b3a3 & 195 & Imatinib & 92 & No & $19+$ \\
\hline 3 & b3a3 & 46 & $\begin{array}{l}\text { Imatinib, } \\
\text { Nilotinib }\end{array}$ & 33 & No & $22+$ \\
\hline 4 & b3a3 & 34 & Imatinib & 30 & No & $77+$ \\
\hline 5 & e8a2 & 107 & $\begin{array}{l}\text { Nilotinib, } \\
\text { Imatinib }\end{array}$ & 93 & No & $5+$ \\
\hline 6 & $\mathrm{e} 19 \mathrm{a} 2$ & 71 & $\begin{array}{l}\text { Imatinib, } \\
\text { Nilotinib }\end{array}$ & 38 & No & $28+$ \\
\hline 7 & $\mathrm{e} 19 \mathrm{a} 2$ & 71 & $\begin{array}{l}\text { Imatinib, } \\
\text { Nilotinib }\end{array}$ & 43 & Yes & 2 \\
\hline
\end{tabular}

TKI = Tyrosine kinase inhibitor; $\mathrm{MMR}=$ Major molecular response; $\mathrm{TFR}=$ Treatment free remission. 
For this study, we defined stable Major Molecular Response (MMR) as an undetectable transcript at nested PCR in all follow-ups in the last 24 months before discontinuation. Molecular monitoring was usually performed every three months during treatment and every month for the first six months after TKI discontinuation, followed by evaluation every six weeks for the remaining six months and every three months after then. ${ }^{8}$

Patients showed a stable MMR, and the median duration of treatment with TKI was 71 months (range: 34-195), the median duration of MMR at nested PCR before discontinuation was 43 months (range: 30-93). Only one patient resumed TKI therapy two months after stopping due to nested PCR positivity in two consecutive controls. The other six patients remained off-treatment at last observation after a median followup of 25 months (range: 5-77). Among these, five patients remained negative, with an undetectable transcript in all samples after discontinuation. Patient 3, who stopped the second line Nilotinib for intolerance, showed a fluctuation after stopping TKI between negative PCR and low-level positivity at the second step of nested PCR (2 out of 13 samples). No progressions occurred. All patients, including the one that resumed therapy, are in MMR at the last follow-up.

Although nowadays nested PCR represents the only routinely accepted method to monitor molecular response in CML patients with atypical transcripts, the qualitative nature of its results is not enough in an era of quantitative analysis. For this reason, we used recently published droplet digital PCR (ddPCR) assays ${ }^{9}$ to quantify the BCR-ABL1 levels in 3 of 6 collected patients in TFR (unfortunately, for 3 of these, RNA samples were not available after routine diagnostic tests). Twenty-one follow-ups were tested after TKI suspension ( 7 for the patient 1, 8 for the patient 2, and 6 for the patient 3), and results were reported in Figure 1 and Table 2.

All the tested follow-ups showed a BCRABL1/ABL1 percentage lower than $0.1 \%$ during all the TFR periods; in some points, \%BCR-ABL1/ABL1 achieve values lower than $0.01 \%$, and in 6 follow-ups BCR-ABL1 levels resulted undetectable $(0 \%)$. Our data in these three patients confirmed with quantitative information the achievement of a stable MMR, previously defined only by qualitative data (nested PCR).

To our knowledge, there are no reports in the literature about patients with atypical transcripts who discontinued therapy. Although current guidelines do not recommend discontinuation for patients lacking a standardized quantitative method for response monitoring, we observed that our small cohort stopped
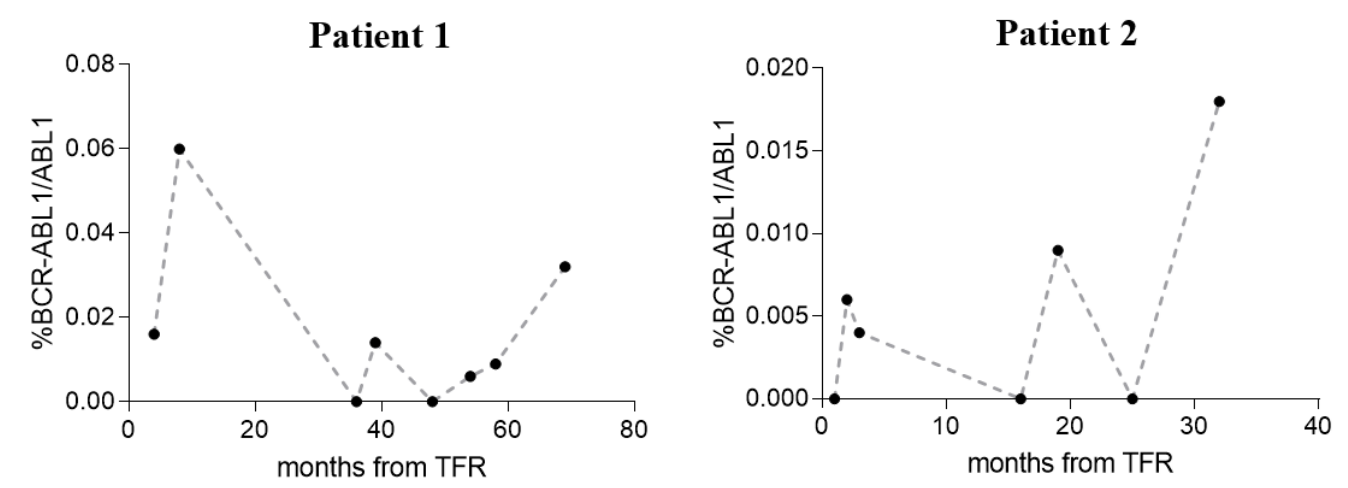

Patient 3

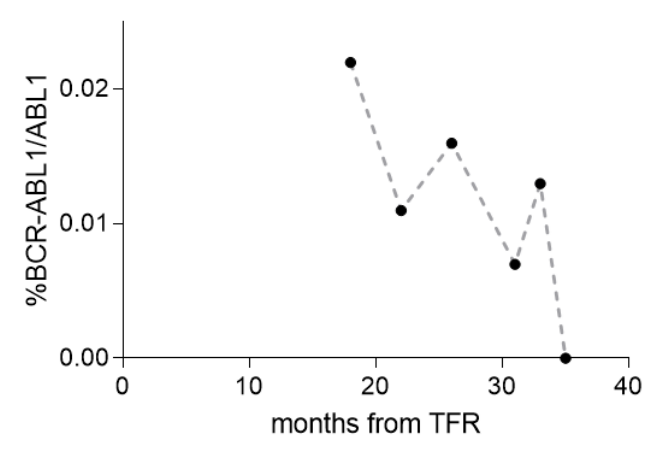

Figure 1: Monitoring by ddPCR of BCR-ABL1 levels in 3 CML patients with atypical transcripts during the treatment-free remission (TFR) phase. Percentage of BCR-ABL1/ABL1 was reported on y-axis, while the time of follow-up after TFR was on $\mathrm{x}$-axis and was indicated in months. 
Table 2. \%BCR-ABL1/ABL1 levels in 3 CML patients with atypical transcript monitored with ddPCR.

\begin{tabular}{|c|c|c|}
\hline Sample & Months after TFR & ddPCR \%BCR-ABL1/ABL1 \\
\hline Patient 1 & 4 & 0.016 \\
\hline Patient 1 & 8 & 0.060 \\
\hline Patient 1 & 36 & 0.000 \\
\hline Patient 1 & 39 & 0.014 \\
\hline Patient 1 & 48 & 0.000 \\
\hline Patient 1 & 54 & 0.006 \\
\hline Patient 1 & 58 & 0.009 \\
\hline Patient 1 & 69 & 0.032 \\
\hline Patient 2 & 1 & 0.000 \\
\hline Patient 2 & 2 & 0.006 \\
\hline Patient 2 & 3 & 0.004 \\
\hline Patient 2 & 16 & 0.000 \\
\hline Patient 2 & 19 & 0.009 \\
\hline Patient 2 & 25 & 0.000 \\
\hline Patient 2 & 32 & 0.018 \\
\hline Patient 3 & 18 & 0.022 \\
\hline Patient 3 & 22 & 0.011 \\
\hline Patient 3 & 26 & 0.016 \\
\hline Patient 3 & 31 & 0.007 \\
\hline Patient 3 & 33 & 0.013 \\
\hline Patient 3 & 35 & 0.000 \\
\hline
\end{tabular}

TFR: Treatment free remission.

the treatment successfully.

In this particular moment where CML care is focused on TKI discontinuation, it seems rather important to us to raise consciousness on the possibility to extend the policy of withdrawing TKI even in carefully selected patients harboring atypical transcripts. The rapid evolution of molecular technologies in the last years, in particular the use of ddPCR, could help the exploration of TFR opportunity also in these rare cases and could pave the way to study how the atypical transcripts affect treatment response.

In our opinion, this leads to two important matters of debate: first, may qualitative analysis suffice, at least in a specific setting, for MRD monitoring? This could be of interest to all low-income countries that cannot afford to perform RT-qPCR during treatment nor discontinuation. Second, is it plausible to assume that patients who carried the atypical transcript may also have the opportunity to stop treatment? Although our cohort is limited, these patients behave as "standard breakpoints carriers" in terms of survival and progression during therapy. Furthermore, among our cases was also present one patient with fluctuation of BCR-ABL1 levels during the TFR phase, which was not at the end associated with relapse. Although the definition of fluctuation cannot be the same of the ASTIM due to the lacking of the MMR threshold to consider, we observed that, as in the mentioned study, the occurring of this pattern of positive values of BCRABL1 did not impair the successfulness of discontinuation. ${ }^{10}$

Although our data are encouraging and represent a preliminary step to consider the possibility of TKI discontinuation also for these patients, further reports are of course needed to make our observations more reliable: the increase in the number of cases we were able to collect, as well as the application of new quantitative technologies, such as digital PCR, for the MRD quantification.

To date, there are no standardized primers and probes set to monitor patients with atypical BCR-ABL1 transcripts with qRT-PCR, thus it is impossible to compare the two methods, and it is difficult to define a priori which is the best technique between qRT-PCR and ddPCR. Based on our experience and literature, ddPCR technology provides absolute quantification of target copies, without the need for standard curves; the massive sample partitioning enables the reliable measurement of small copy numbers of transcript, and error rates are reduced by removing the amplification efficiency reliance of qRT-PCR. Furthermore, recently published works, that compare qRT-PCR and ddPCR methods for the monitoring of canonical BCR-ABL1 fusion transcripts, suggest that ddPCR could be a reliable and promising tool and conclude that ddPCR has a good agreement with qRT-PCR, but it is more 
precise and reproducible in the quantification of very low BCR-ABL1 transcript levels. ${ }^{11-14}$ Lastly, a standardization process of BCR-ABL1 molecular monitoring for CML patients with rare variants by harmonization to an International Scale could be useful to define MRD levels better, compare results, and establish a better therapeutic strategy.

Matteo Dragani ${ }^{1 *}$, Jessica Petiti ${ }^{1}$, Giovanna Rege-Cambrin ${ }^{1}$, Enrico Gottardi ${ }^{1}$, Filomena Daraio ${ }^{1}$, Giovanni Caocci ${ }^{2}$, Chiara Aguzzi $^{3}$, Elena Crisà ${ }^{4}$, Giacomo Andreani ${ }^{1}$, Francesca Caciolli ${ }^{1}$ and Carmen Fava ${ }^{1}$.

${ }^{1}$ Department of Clinical and Biological Sciences, University of Turin, Orbassano.

${ }^{2}$ Department of Hematology, University of Cagliari, Cagliari.

${ }^{3}$ Department of Biotechnologies and Hematology, University of Turin, Turin.

${ }^{4}$ Department of Hematology, University of Oriental Piedmont, Novara.

Competing interests: The authors declare no conflict of Interest.

Correspondence to: Matteo Dragani. Department of Clinical and Biological Sciences, University of Turin, Regione Gonzole 10, 10043 Orbassano (TO). Tel.: +393452190280. E-mail: matteo.dragani@gmail.com

\section{References:}

1. Baccarani M, Castagnetti F, Gugliotta G, et al. The proportion of different BCR-ABL1 transcript types in chronic myeloid leukemia. An international overview. Leukemia. May 2019;33(5):1173-1183. https://doi.org/10.1038/s41375-018-0341-4 PMid:30675008

2. Pfirrmann M, Evtimova D, Saussele S, et al. No influence of BCR-ABL1 transcript types e13a2 and e14a2 on long-term survival: results in 1494 patients with chronic myeloid leukemia treated with imatinib. Journal of cancer research and clinical oncology. May 2017;143(5):843-850. https://doi.org/10.1007/s00432-016-2321-2 PMid:28083711

3. Jain P, Kantarjian H, Patel KP, et al. Impact of BCR-ABL transcript type on outcome in patients with chronic-phase CML treated with tyrosine kinase inhibitors. Blood. Mar 10 2016;127(10):1269-75. https://doi.org/10.1182/blood-2015-10-674242

PMid:26729897 PMCid:PMC4786836

4. Castagnetti F, Gugliotta G, Breccia M, et al. The BCR-ABL1 transcript type influences response and outcome in Philadelphia chromosomepositive chronic myeloid leukemia patients treated frontline with imatinib. American journal of hematology. Aug 2017;92(8):797-805. https://doi.org/10.1002/ajh.24774 PMid:28466557

5. Pagnano KBB, Miranda EC, Delamain MT, et al. Influence of BCR-ABL Transcript Type on Outcome in Patients With Chronic-Phase Chronic Myeloid Leukemia Treated With Imatinib. Clinical lymphoma, myeloma \& leukemia. Nov 2017;17(11):728-733. https://doi.org/10.1016/j.clml.2017.06.009 PMid:28822797

6. Hochhaus A, Saussele S, Rosti G, et al. Chronic myeloid leukaemia: ESMO Clinical Practice Guidelines for diagnosis, treatment and followup. Annals of oncology : official journal of the European Society for Medical Oncology. Jul 1 2017;28(suppl 4):iv41-iv51. https://doi.org/10.1093/annonc/mdx219

7. Radich JP, Deininger M, Abboud CN, et al. Chronic Myeloid Leukemia Version 1.2019, NCCN Clinical Practice Guidelines in Oncology. Journal of the National Comprehensive Cancer Network : JNCCN. Sep 2018;16(9):1108-1135.

https://doi.org/10.6004/jnccn.2018.0071

PMid:30181422

8. van Dongen JJ, Macintyre EA, Gabert JA, et al. Standardized RT-PCR analysis of fusion gene transcripts from chromosome aberrations in acute leukemia for detection of minimal residual disease. Report of the BIOMED-1 Concerted Action: investigation of minimal residual disease in acute leukemia. Leukemia. Dec 1999;13(12):1901-28. https://doi.org/10.1038/sj.leu.2401592 PMid:10602411

9. Petiti J, Lo Iacono M, Dragani M, et al. Novel Multiplex Droplet Digital PCR Assays to Monitor Minimal Residual Disease in Chronic Myeloid Leukemia Patients Showing Atypical BCR-ABL1 Transcripts. Journal of clinical medicine. May 13 2020;9(5) https://doi.org/10.3390/jcm9051457 PMid:32414125 PMCid:PMC7290999

10. van Dongen JJ, Macintyre EA, Gabert JA, et al. Standardized RT-PCR analysis of fusion gene transcripts from chromosome aberrations in acute leukemia for detection of minimal residual disease. Report of the BIOMED-1 Concerted Action: investigation of minimal residual disease in acute leukemia. Leukemia. Dec 1999;13(12):1901-28 https://doi.org/10.1038/sj.leu.2401592 PMid:10602411

11. Bernardi, S. et al. "Variant-specific discrepancy when quantitating BCRABL1 e13a2 and e14a2 transcripts using the Europe Against Cancer qPCR assay." Is dPCR the key? European journal of haematology 103, 272-273,

https://doi.org/10.1111/ejh.13282

PMid:31233644

12. Chung, H. J. et al. Performance Evaluation of the QXDx BCR-ABL \%IS Droplet Digital PCR Assay. Ann Lab Med 40, 72-75, https://doi.org/10.3343/alm.2020.40.1.72 PMid:31432643 PMCid:PMC6713652

13. Fava, C. et al. A Comparison of Droplet Digital PCR and RT-qPCR for BCR-ABL1 Monitoring in Chronic Myeloid Leukemia. Blood 134, 2092 2092 ,

https://doi.org/10.1182/blood-2019-125614

14. Franke, G. N. et al. Comparison of Real-Time Quantitative PCR and Digital Droplet PCR for BCR-ABL1 Monitoring in Patients with Chronic Myeloid Leukemia. The Journal of molecular diagnostics: JMD 22, 8189 ,

https://doi.org/10.1016/j.jmoldx.2019.08.007 PMid:31669230 